\title{
Enhancement of photocatalytic property on ZnS/MoS2 composite under visible light irradiation
}

\author{
Jiushan Cheng ${ }^{1,2}$, Lu Han ${ }^{1}$, Yen Wei ${ }^{2}$ and Qiang Chen ${ }^{1}$ \\ ${ }^{1}$ Laboratory of Plasma Physics and Materials, Beijing Institute of Graphic Communication, Beijing 102600, China \\ ${ }^{2}$ Department of Chemistry and the Tisnghua Center for Frontier Polymer Research, Tsinghua University, Beijing, 10084, China
}

\begin{abstract}
In this paper, the composite $\mathrm{ZnS} / \mathrm{MoS}_{2}$ was obtained via two steps including solvothermal methods. The as-synthesized sample was characterized by X-ray diffraction (XRD), scanning electron microscopy (SEM) and UV-Vis. diffuse reflectance spectra (DRS). The photocatalytic activity of the product was evaluated through photocatalytic degradation of Rhodamine B (Rh B) under UV-Vis. light irradiation; the electrical conductivity of $\mathrm{ZnS} / \mathrm{MoS}_{2}$ composites was significantly improved compared to $\mathrm{ZnS}, \mathrm{MoS}_{2}$, respectively. The results showed that the $\mathrm{ZnS} / \mathrm{MoS}_{2}$ composite photocatalyst possesses better photocatalytic activity in degrading Rh $\mathrm{B}$ than the single $\mathrm{ZnS}$ or the single $\mathrm{MoS}_{2}$. The better photocatalytic properties may be due to the synergetic effect of two semiconductors, because of which electrons and holes were separated effectively. And its specific microstructure played an active role in evaluating photocatalytic performance.
\end{abstract}

\section{Introduction}

Environmental degradation is one of the challenging phenomenons due to the increasing rate of the industrialization in the world wide. Especially, organic dyes have been wildly used in the plastic, paper, textile, and other chemical industries in our lives [1-3]. And it can cause environmental pollution.

However, photocatalysis is one of the promising methods to remove the organic components from waste water. Semiconductor photocatlysts have attracted a subject of extensive interest due to their fundamental importance applications for solving energy and pollution problems. Recently, molybdenum disulfide $\left(\mathrm{MoS}_{2}\right)$, shows great potential for visible light irradiated photocatalysis applications. More and more researchers have been extensively investigated $\mathrm{MoS}_{2}$ as a promising candidate for many materials, due to its excellent electrical carrier mobility, high chemical reactivity and optical properties [4-7]. For instance, Xiuquan Gu [8] et al. obtained $\mathrm{MoS}_{2} / \mathrm{Ag}_{3} \mathrm{PO}_{4}$ hybrids by incorporating $\mathrm{MoS}_{2}$ nanosheets during the preparation process of $\mathrm{Ag}_{3} \mathrm{PO}_{4}$ microparticles. The paper pointed out that the photocatalytic activity of $\mathrm{MoS}_{2} / \mathrm{Ag}_{3} \mathrm{PO}_{4}$ hybrid got improved owning to compositing with trace amounts $\mathrm{MoS}_{2}$. The group of Aiyong Zhang [9] prepared layer-controlled $\mathrm{MoS}_{2}$ on three-dimensional $\mathrm{Bi}_{2} \mathrm{~S}_{3}$ micro-flower through sacrificial templating strategy. The paper showed that 3D flower-like hybrid nanostructures make $\mathrm{MoS}_{2} / \mathrm{Bi}_{2} \mathrm{~S}_{3}$ to display great photocatalysis under visible light irradiation.

Similarly, among the plenty of metal sulfide photocatalysts, especially, $\mathrm{ZnS}$, have been paid more attention from the scientific research owing to their optical properties and unique catalytic functions. However, $\mathrm{ZnS}$ is responsive only in ultraviolet light region. To address this issue, many means have been used, such as nanomaterials morphology modification and semiconductor compositing. These methods are proved to be effective, and the best way is meeting the energy demands of $\mathrm{ZnS}$ with wide band-gap to maintain the catalytic activity [10-11]. Longxin $\mathrm{Hu}$ [12] et al. reported that $\mathrm{SnO}_{2} / \mathrm{ZnS}$ nanocomposite was prepared by one step hydrothermal method. It was found that the compound showed the great photocatalytic activity. The photocatalytic degradation of $\mathrm{RhB}$ (Rhoadmine B) reached $95 \%$ at given time. In Baibiao Huang's group [13], they prepared $\mathrm{ZnS}$ microspheres with Mn-doping by a facile solvothermal route. And it was found that Mn-doping ZnS exhibited better photocatalytic performance than pure $\mathrm{ZnS}$.

Owing to there have been few studies related to $\mathrm{ZnS} / \mathrm{MoS}_{2}$ composite. In this paper, we successfully prepared semiconductor of $\mathrm{MoS}_{2}$ decroated with $\mathrm{ZnS}$ as catalyst for efficient degradation of dye. Therefore, we pay attention to the $\mathrm{ZnS}$ nanoparticles coated $\mathrm{MoS}_{2}$ nanosheets system connecting two chemically stable and inexpensive multifunctional semiconductor materials together. In this system, $\mathrm{MoS}_{2}$ nanosheets work as narrow band gap p-type semiconductor and $\mathrm{ZnS}$ serves as wide band-gap n-type semiconductor. The experimental reagents are simple and easy to get and the reaction conditions are simple and controllable. Experiment results indicate that $\mathrm{ZnS}$ composite $\mathrm{MoS}_{2}$ exhibit superior photocatalyst performance towards $\mathrm{RhB}$. 


\section{Experimental}

In a typical procedure, $1.5 \mathrm{mmol} \mathrm{Na}_{2} \mathrm{MoO}_{4}$ and $15 \mathrm{mmol}$ $\mathrm{C}_{3} \mathrm{H}_{7} \mathrm{NO}_{2} \mathrm{~S}$ were dissolved in $80 \mathrm{ml}$ deionized water. Then, the solution was transferred into a Teflon-lined stainless-steel autoclave, which was sealed and heated at $220^{\circ} \mathrm{C}$ for $24 \mathrm{~h}$, and then cool down to room temperature. The as-prepared precipitates were washed by ethyl alcohol and distilled water three times respectively. Then the black powder was collected and dried in vacuum at $80^{\circ} \mathrm{C}$ for $12 \mathrm{~h}$. The black precipate was denoted as $\mathrm{MoS}_{2}$. $0.1 \mathrm{~g}$ thioacetamide $\left(\mathrm{CH}_{3} \mathrm{CSNH}_{2}\right), 0.6758 \mathrm{~g}$ zinc acetate $\left(\mathrm{Zn}\left(\mathrm{CH}_{3} \mathrm{COO}\right)_{2}\right)$ and $0.245 \mathrm{~g}$ Sodium Sulfide $\left(\mathrm{Na}_{2} \mathrm{~S}\right)$ were dissolved in $20 \mathrm{ml}$ deionized water. The mixture was full stirred for $2 \mathrm{~h}$ at room temperature. Then, the solution was transferred into a Teflon-lined stainless-steel autoclave, which was sealed and heated at $170^{\circ} \mathrm{C}$ for $24 \mathrm{~h}$, and then cool down to room temperature. Then, the precipitates were collected and washed by ethyl alcohol and distilled water three times respectively. Then obtained powder was dried in vacuum at $60^{\circ} \mathrm{C}$ for $12 \mathrm{~h}$. The final powder was called $\mathrm{ZnS}$.

0.1 as-prepared $\mathrm{MoS}_{2}$, and $0.2 \mathrm{~g}$ Sodium dodecyl sulfate(SDS), $1.3516 \mathrm{~g}$ zinc acetate, $0.49 \mathrm{~g}$ Sodium Sulfide were dissolved in $60 \mathrm{ml}$ deionized water. Then, the solution was transferred into a Teflon-lined stainless-steel autoclave, which was sealed and heated at $170^{\circ} \mathrm{C}$ for $2 \mathrm{~h}$, and then cool down to room temperature. Then, the precipitates were collected and washed by ethyl alcohol and distilled water three times respectively. Then obtained powder was dried in vacuum at $60^{\circ} \mathrm{C}$ for $12 \mathrm{~h}$ which denoted as $\mathrm{ZnS} / \mathrm{MoS}_{2}$.

The as-prepared products were characterized by powder X-ray diffraction (XRD) in Bruker D8 diffractometer using $\mathrm{Cu} K \alpha$ radiation. The surface morphology of the samples were observed by a HITACHI S3500 scanning electron microscopy (SEM). The light absorption properties were measured using UV-Vis. diffuse reflectance spectrophotometer U-4100 (DRS) with a wavelength range of $300 \sim 800 \mathrm{~nm}$.

The photocatalytic activity of the samples was determined by degradation of Rhoadmine $\mathrm{B}(\mathrm{RhB})$ in an aqueous solution under visible light irradiation. The photo degradation experiments were carried out under UV-Vis. light irradiation whose source was a $300 \mathrm{~W}$ Xe lamp. The distance between the xenon lamp and the sample was about $50 \mathrm{~cm}$. In the each experiment, $20 \mathrm{mg}$ photocatalyst was added into $20 \mathrm{~mL} \mathrm{RhB}$ solution $\left(\mathrm{C}_{0}=10 \mathrm{mg} / \mathrm{L}\right)$. Prior to irradiation, the suspension was sonicated for a few minutes and then magnetically stirred in dark for hours to obtain desorption-adsorption equilibrium. At given time intervals, $5 \mathrm{~mL}$ of the sample solution was collected and separated through centrifugation. The HITACHI U-4100 UV-Vis. spectrometer was used to determine the concentration of RhB solution during the photocatalytic degradation process.

The electrochemical properties of all the samples were studied using electrochemical workstation (PGZ402, VoltaLab, France) with a three-electrodecon figuration. The samples were then used to modify screen-printed carbon electrodes (SPCEs) to immobilize antibodies and enhance the electrochemical signals. $1 \mu 1$ of $1 \mathrm{mg} / \mathrm{ml}$ $\mathrm{ZnS} 、 \mathrm{MoS}_{2}$ and $\mathrm{ZnS} / \mathrm{MoS}_{2}$ were spread on the working area of SPECs using a micropipette and the film was dried at room temperature, resepectively. All the cyclic voltammetry $(\mathrm{CV})$ measurements were recorded in $0.1 \mathrm{M}$ phosphate buffer (PBS, pH7.4) containing $5 \mathrm{mM}$ $\mathrm{K}_{3}\left[\mathrm{Fe}(\mathrm{CN})_{6}\right]$ and $\mathrm{K}_{4}\left[\mathrm{Fe}(\mathrm{CN})_{6}\right]$ in the potential range of -1000 to $+1000 \mathrm{mV}$. A reproducible voltammogram was obtained under steady-state conditions after about five cycles. All the electrochemical impedance spectroscopy (EIS) measurements were carried out in $0.01 \mathrm{M}$ PBS containing $5 \mathrm{mM} \mathrm{K} \mathrm{K}_{3}\left[\mathrm{Fe}(\mathrm{CN})_{6}\right]$ and $\mathrm{K}_{4}\left[\mathrm{Fe}(\mathrm{CN})_{6}\right]$ in a frequency range from 0.1 to $104 \mathrm{~Hz}$ at $220 \mathrm{mV}$.

\section{Results and discussion}

The XRD patterns of the samples are presented in Figure. 1. There are no other phases and peaks of impurities, demonstrating that all the samples are pure. $\mathrm{MoS}_{2}$ and $\mathrm{ZnS}$ possess the phases corresponding to JCPDS No. $37-1492(\mathrm{a}=3.161 \AA$ and $\mathrm{b}=3.161 \AA, \mathrm{c}=12.299 \AA)$ and 65-5476 $(\mathrm{a}=5.404 \AA, \mathrm{b}=5.404 \AA$, and $\mathrm{c}=5.404 \AA)$ respectively. The XRD pattern of the $\mathrm{ZnS} / \mathrm{MoS}_{2}$ nanohybrid shows $\mathrm{MoS}_{2}$ major peaks at corresponding to

(100), (103) , (110) and there are peaks, like (111)、（220）、（311） observed related to $\mathrm{ZnS}$. And, no other phase is detected in the $\mathrm{ZnS} / \mathrm{MoS}_{2}$ composite before and after the photocatalytic degradation, suggesting that the composite is stable and there is no chemical reaction between $\mathrm{MoS}_{2}$ and $\mathrm{ZnS}$.

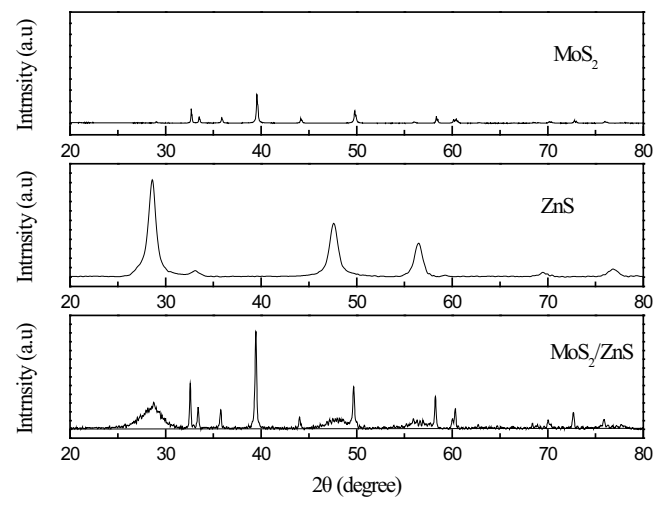

Figure 1. XRD pattern of the samples $\mathrm{MoS}_{2}, \mathrm{ZnS}, \mathrm{ZnS} / \mathrm{MoS}_{2}$.

The morphologies of the pure $\mathrm{MoS}_{2}, \mathrm{ZnS}$ and $\mathrm{ZnS} / \mathrm{MoS}_{2}$ composite are observed by SEM. Figure.2a exhibits a representative SEM image of pure $\mathrm{MoS}_{2}$. The highly monodispersed rough ball-like spheres are obtained which is composed of thin plates as shown in Figure. $2 \mathrm{~b}$. The nanoplates align radically and tightly to assemble into the uniform spheres. The surfaces of nanoplates are smooth. The SEM micrograph of $\mathrm{ZnS}$ in Figure2c show a large number of plates arranged irregularly together. It can be seen the shapes of plate is the block shaped and the length is about $1 \mu \mathrm{m}$ on average. Regarding to $\mathrm{ZnS} / \mathrm{MoS}_{2}$ composite, it could be seen that the microspheres are organized by hundreds of thin nanoplates and some $\mathrm{ZnS}$ plates are together with 
sphere with a diameter of $1 \mu \mathrm{m}$ (Figure. $2 \mathrm{~d})$.

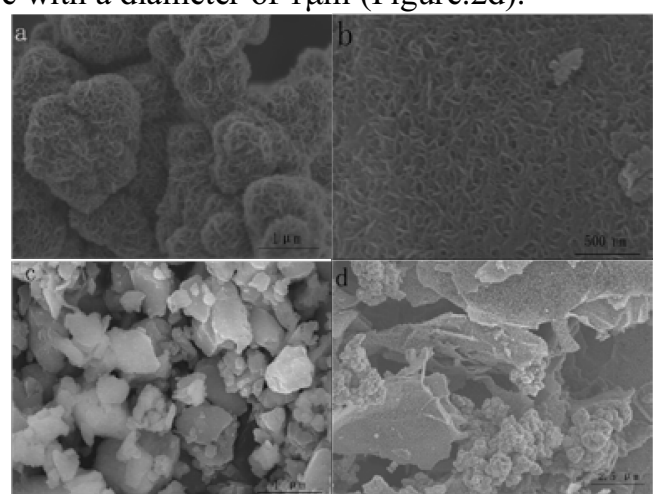

Figure 2. The SEM images of samples (a,b) $\mathrm{MoS}_{2}$, (c) ZnS, (d) $\mathrm{ZnS} / \mathrm{MoS}_{2}$

The absorption property is one of the most important properties to characterize the optical property of semiconductor. Figure. 3 shows the UV-Vis. diffuse reflectance spectra of $\mathrm{ZnS}$ and $\mathrm{ZnS} / \mathrm{MoS}_{2}$ composite. It could be seen that the pure $\mathrm{ZnS}$ has an absorption edge occurred at about $375 \mathrm{~nm}$. The absorption edge of the pure $\mathrm{ZnS} / \mathrm{MoS}_{2}$ is approximately at $390 \mathrm{~nm}$. From the picture inset Figure.3, it can be seen UV-Vis. diffuse reflectance spectra of $\mathrm{MoS}_{2}$. The absorption of $\mathrm{ZnS} / \mathrm{MoS}_{2}$ composite during visible light range is much improved may be owing to $\mathrm{MoS}_{2}$ which plays an important role.

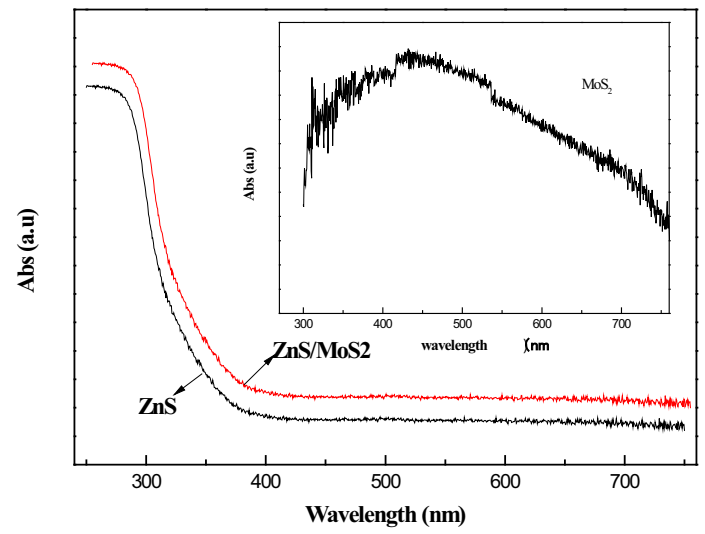

Figure 3. UV-Vis. DRS spectra of the samples $\mathrm{ZnS}, \mathrm{ZnS} / \mathrm{MoS}_{2}$, the inset of Figure is UV-Vis. DRS of sample $\mathrm{MoS}_{2}$.

The photocatalytic activities of as-prepared samples were evaluated by degrading Rhodamine B ( Rh B) under $\mathrm{UV}-\mathrm{Vis}$. light irradiation. The photodegradation of the $\mathrm{RhB}$ as a function of the irradiation time over $\mathrm{ZnS} / \mathrm{MoS}_{2}$ composite is presented in Figure 4. We can see there is no significant change in the concentration curve with time when the photocatalysis is absent, which means $\mathrm{Rh} B$ is very stable without photocatalysis only under UV-Vis. light. From the curves of the concentration changes of RhB dye, it can be seen that the photocatalytic degradation rate of all as-prepared samples are increased with the increasing of the reaction time. In 80 minutes, nearly $70 \% \mathrm{RhB}$ is degraded by $\mathrm{ZnS} / \mathrm{MoS}_{2}$. whereas in 80 minutes, the $\mathrm{MoS}_{2}$ and $\mathrm{ZnS}$ degraded the RhB concentration by $64 \%$, and $82 \%$, respectively. It could be seen that the $\mathrm{ZnS} / \mathrm{MoS}_{2}$ composite exhibits the best photocatalytic activity among the samples.

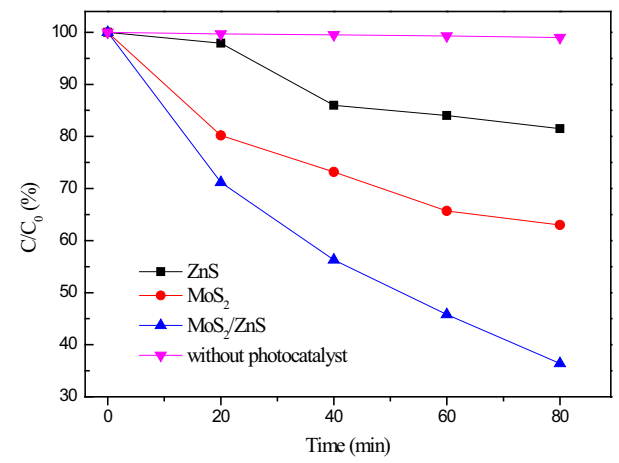

Figure 4. Photocatalytic degradation efficiency of the Rh.B over no photocatalyst, $\mathrm{MoS}_{2}, \mathrm{ZnS}, \mathrm{ZnS} / \mathrm{MoS}_{2}$.

In order to further confirm the enhanced conductivity of $\mathrm{ZnS} / \mathrm{MoS}_{2}$, CV curves of $\mathrm{ZnS} / \mathrm{MoS}_{2}, \mathrm{ZnS}, \mathrm{MoS}_{2}$ were measured in $0.1 \mathrm{M}$ phosphate buffer $(\mathrm{pH}$ 7.2) containing $5 \mathrm{mM} \quad \mathrm{K}_{3}\left[\mathrm{Fe}(\mathrm{CN})_{6}\right]$ and $\mathrm{K}_{4}\left[\mathrm{Fe}(\mathrm{CN})_{6}\right]$ as electrolyte. In Figure.5 a pair of symmertric and well-separated redox peaks was observed, indicating that the measured electroconductivity was based on the redox mechanism [14-17]. It was also noted that $\mathrm{ZnS} / \mathrm{MoS}_{2}$ had another cathodic peak at $-700 \mathrm{mV}$ may due to the redox of $\mathrm{MoS}_{2}$. $\mathrm{ZnS} / \mathrm{MoS}_{2}$ exhibited higher current density than single $\mathrm{ZnS}$ and $\mathrm{MoS}_{2}$ due to the accelerated electron transfer by $\mathrm{MoS}_{2}$. This observation indicates an irreversible electrochemical reduction of $\mathrm{MoS}_{2}$ when CV is conducted in the inorganic electrolyte at the potential range from 1000 to $-1000 \mathrm{mV}$. It means that the $\mathrm{MoS}_{2}$ was successfully reduced.

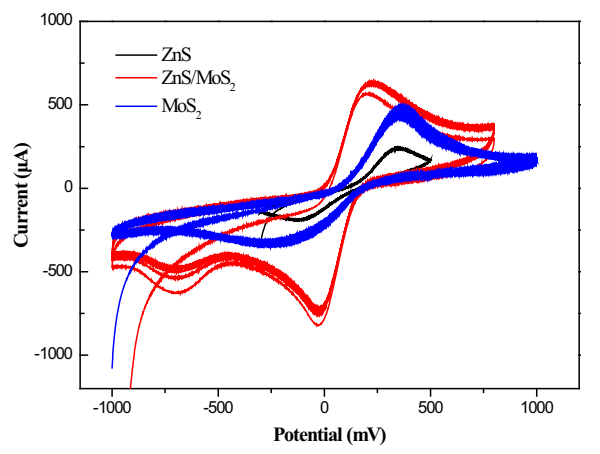

Figure 5. $\mathrm{CV}$ curves of $\mathrm{MoS}_{2}, \mathrm{ZnS}, \mathrm{ZnS} / \mathrm{MoS}_{2}$ at the scan rate of $100 \mathrm{mV} \mathrm{s} 1$ in $0.1 \mathrm{M}$ PBS (pH 7.4) at RT.

It is obvious that $\mathrm{ZnS} / \mathrm{MoS}_{2}$ composite nanostructures possess more powerful photocatalytic capacity for the degradation of $\mathrm{Rh} \mathrm{B}$. As a consequence, a possible mechanism is proposed. According to some papers [18-21], we deduce that $\mathrm{MoS}_{2}$ and $\mathrm{ZnS}$ have matching band potential.

Under irradiation, the photocatalyst could generate electrons and holes. The conduction band and valence band edge positions of $\mathrm{MoS}_{2}$ are associated with those of $\mathrm{ZnS}$, so both electrons and holes are transferred to $\mathrm{MoS}_{2}$. Holes transfer to $\mathrm{MoS}_{2}$ nanoflakes, which increases charge separation. Thus, $\mathrm{MoS}_{2}$ acts as an oxidation site. In another words, the band potentials of both $\mathrm{MoS}_{2}$ and $\mathrm{ZnS}$ 
semiconductors are conductive to prevent the recombination of electrons and holes in the $\mathrm{ZnS} / \mathrm{MoS}_{2}$ composite. In general, the $\mathrm{ZnS} / \mathrm{MoS}_{2}$ composite with special nanostructure and interface increases the visible light absorption and restrains the recombination of electron-hole pairs.

\section{Conclusion}

In summary, $\mathrm{ZnS} / \mathrm{MoS}_{2}$ composite photocatalyst was got by two steps which in order were solvothermal methods. The microsphere of the composite was composed of hundreds of $\mathrm{MoS}_{2}$ thin nanoplates and some $\mathrm{ZnS}$ plates were together with the sphere about a diameter of $1 \mu \mathrm{m}$ from the view of morphology. From the photodegradation of the $\mathrm{Rh} \mathrm{B}$ organic dye we found that the $\mathrm{ZnS} / \mathrm{MoS}_{2}$ composited photocatalyst displayed better photocatalytic activity than both single $\mathrm{MoS}_{2}$ and single $\mathrm{ZnS}$. As a proof of concept, the $\mathrm{ZnS} / \mathrm{MoS}_{2}$ with good conductivity, superior electron transfer rate and high electrochemical activity is successfully used to detect photocatalytic property.

\section{Acknowledge}

This work was financially supported by a project founded by Beijing Institute of Graphic Communication (No. 27170115005/020), Project supported by Beijing Postdoctoral Research Foundation (No. 10000200272), Beijing college students' scientific research and entrepreneurial action plan (No. 22150116005/046).

\section{References}

1. Ying Wang, Mingxuan Sun, Yalin Fang, Shanfu Sun, Jia He, J. Mater. SCI, 51, 7792016

2. Shaohui Gao, Xuanhua Li, Jinmeng Zhu, Tengteng Tong, ingqing Wei, Small, 12, 56922016

3. Shijing Liang, Zhouming Zhou, Xiuqin Wu, Shuying Zhu, Jinhong Bi, Limin Zhou, Minghua Liu, Ling Wu, Molecules, 21. 2132016

4. M.Nath, A. Govindaraj, C.N.R. Rao, , Adv Mater, 13, 283,2001

5. Lei Huang, Xiuli Wang, Jinhui Yang,Gang Liu, Jingfeng Han, Can Li, J Phys Chem C, 11711584 2013

6. Guoping Chen, Dongmei Li, Fan Li, Yuzun Fan,
Haofei Zhao, Yanhong Luo, Richeng Yu, Qingbo Meng, Appl Catal A-Gen, 443-444, 1382012

7. Xiaojie Xu, Linfeng $\mathrm{Hu}$, Nan Gao, Shaoxiong Liu, Swelm Wageh, Ahmed A. Al-Ghamdi, Ahmed Alshahrie, Xiaosheng Fang, Adv Functi Mater, 25, 4452015

8. Shengping Li, Xiuquan Gu,Yulong Zhao, Yinghuai Qiang, Shuang Zhang, Mrirong Sui, J Mater SciMater EL, 27, 3862016

9. Lulu Long, Jiejie Chen, Xing Zhang, Aiyong Zhang, Yuxi Huang, Qing Rong, Hanqing Yu, NPG Asia Materials, 8, doi:10.1038/am/2016.46

10. S.V Prabhakar Vattikuti, Chan Byon, Sora Jeon, Physica B, 502, 1032016

11. Ch. Venkate Reddy, Jaesool Shim, Migyung Cho, J Phy Chem Solids, doi.org/10.1016/j.jpcs.2016.12.011

12. Longxing $\mathrm{Hu}$, Feiyan Chen, Pengfei Hu, Lianpei Zou, Xing Hua, J Mol Catal A-Chem, 4112032016

13. Lu Wang, Peng Wang, Baibiao Huang, Xiaojuan Ma, Gang Wang, Ying Dai, Xiaoyang Zhang, Xiaoyan, Qin, Appl Surf Sci, 391, 5572017

14. Lu Han, Chengmei Liu, Shilei Dong, Caixia Du, Xiaoyong Zhang, Luhai Li, Yen Wei, Biosens Bioelectron 87, 4662017

15. Zhebo Chen, Dustin Cummins, Benjamin N. Reinecke, Ezra Clark, Mahendra K. Sunkare, Thomas F. Jaramill, Nano Lett, 1141682011

16. Jun Chen, Nobuhiro Kuriyama, Huatang Yuan, Hiroyuki T. Takeshita, Tetsuo Sakai, J Am Chem Soc, 123118132001

17. Shixin Wu, Zhiyuan Zeng, Qiyuan He, Zhjuan Wang, Shijie Wang, Yaping Du, Zongyou Yin, Xuping Sun, Wei Chen, Hua Zhang, Small, 8, 22642012

18. Nan Tian, Zhen Li, Danyun Xu, Yang Li, Wenchao Peng, Guoliang Zhang, Fengbao Zhang, Xiabin Fan, Industrial \& Engineering Chemistry Reseach, 55, 87262016

19. Herme G. Baldovi, Marcos Latorre-Sancez, Ivan Esteve-Adell, Anish Khan, Abdullah M. Asiri, Samia A. Kosa, Hermenegildo Garcia, J Nanopart Res, 18, 2402016

20. Quanjun Xiang, Jiaguo Yu, Mietek Jaroniec, J Am Chem Soc, 134, 65752012

21. Xu Zong, Hongjian Yan, Guopeng Wu, Guijun Ma, Fuyu Wen, Lu Wang, Can Li, J Am Chem Soc, 130, 71762008 